\title{
Time Probabilistic Vehicle Optimal Route using Ant Colony Algorithm
}

\author{
Ming $\mathrm{Fu}^{\mathrm{a}}$, Jian Zhou ${ }^{\mathrm{a}, *}$, Lifang Wang ${ }^{\mathrm{b}}$ \\ ${ }^{a}$ School of management science and engineering, Anhui University of Finance \&Economics, Bengbu 233030, Anhui, China \\ ${ }^{b}$ School of international trade and economics, Anhui University of Finance\&Economics, Bengbu 233030, Anhui, China
}

\begin{abstract}
Vehicle routing problem is one of hot issues in the past decade; the paper proposes an important variant of vehicle routing problem, which we name time probabilistic vehicle routing problem. We find the time spent on the road from point A to point B always changes because of dynamic road condition, dynamic weather et al. However, the probability is certain that the vehicle can travel from point A to Point B within certain time intervals. Ant colony algorithm is an efficient distributed algorithm, and has been widely used in various fields. The ant colony algorithm is introduced to obtain the optimal solution by observing the characteristics of the problem. The Ants also search for the next transfer node according to probability in ant colony algorithm. Ant colony algorithm can solve this kind of problem effectively.
\end{abstract}

Keywords: ant colony algorithm; vehicle routing problem; swarm algorithm

(Submitted on April 29, 2017; Revised on August 12, 2017; Accepted on September 23, 2017)

(C) 2017 Totem Publisher, Inc. All rights reserved.

\section{Introduction}

The vehicle routing problem (VRP) is one of the most significant subjects for logistic activities [1]. The main target of vehicle routing problem is to get the least cost routes for fleet vehicles departing from one depot to a set of service points. The cost mainly includes the length of time and distance [2]. Each route starts from the depot and also finishes at the depot in the end.

The problem was first proposed by Dantzig and Ramser in 1959; there is already several researches on various VRP problems in the literature over the past decade [12]. More complex constraints were put forward gradually in the following research, such as time windows or limited capacity of vehicles. Several variants of the VRP were proposed for this reason. We briefly introduce several important VRP varants in the following content.

The capacitated vehicle routing problem (CVRP) is one of important variants where vehicles have limited capacities. One vehicle cannot serve all customers on one trip. The multi-depot vehicle routing problem (MDVRP) is another typical variant of the VRP. The main character is that there is not only one depot in such model, each vehicle can depart from one depot and return back to any depot after serving customers. The periodic vehicle routing problem (PVRP) is also a classic variant of the VRP; this type of problem provides time windows in which the customers are available to receive service of the vehicle. The vehicle routing problem with pick-ups and deliveries (VRPPD) is also an extension of the VRP where some customers require pickup service while other customers expect delivery service of the vehicle [11].

The time which the vehicle travels from point A to B is certain in all the VRP model and the VRP variants model mentioned above, but in fact the time spent on the road from point A to B is usually not a constant value in actual life because of dynamic road congestion, different fatigue degree of driver and the vehicle condition etc. In actual life, the time consumed on the road is always expressed through probability, such as the probability of passing from point A to B within 10 minutes is 0.65 , while the probability of passing from point A to B within 20 minutes is 0.95 . The more time spend on the road, the greater the passing probability is [9]. 


\section{The problem description and solutions analysis}

The paper is trying to define a new variant of the VRP. The new variant of the VRP is the time probabilistic vehicle routing problem (TPVRP), which will be further illustrated by a simple example in the following content. Through the simple example, let us have an intuitive understanding of TPVRP [10]. The complete and complex mathematical model will be set up in the next section.

Firstly, we describe the model and make the following assumptions. These are one depot and their customers, and there is only one vehicle available at the depot at the beginning. The vehicle has a capacity big enough to serve all customers on one trip. In the figure below, the node 1 and 5 represent the same depot, the node 5 is the mirror image of node 1 , while the node 2, 3 and 4 represent customers. The vehicle departs from node 1 and return back to node 5 in the end after serving all customers. So, there are six different routes because of there are only one vehicle, there will be more solutions if there are multiple vehicles. So, the solutions are 1-2-3-4-5, 1-2-4-3-5, 1-3-2-4-5, 1-3-4-2-5, 1-4-2-3-5, and 1-4-3-2-5, which are showed in Figure 2 to Figure 7 . But which is the optimal route in this simple model?

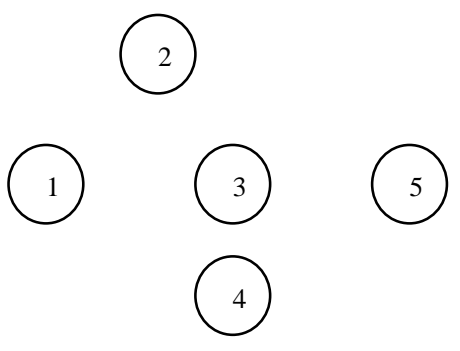

Figure 1. The figure of position distribution

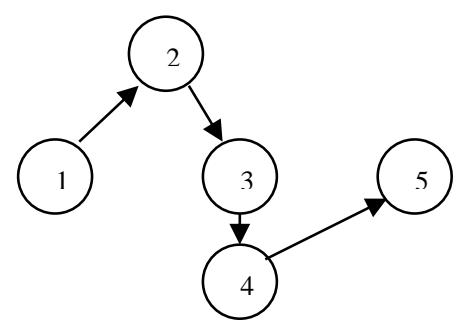

Figure 2. The figure of the first route

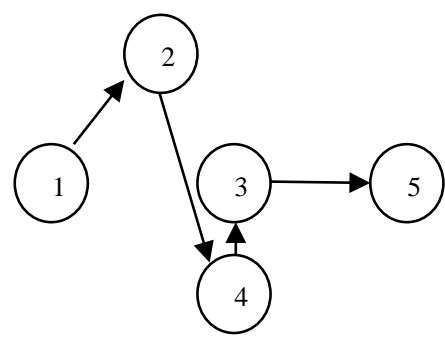

Figure 3. The figure of the second route

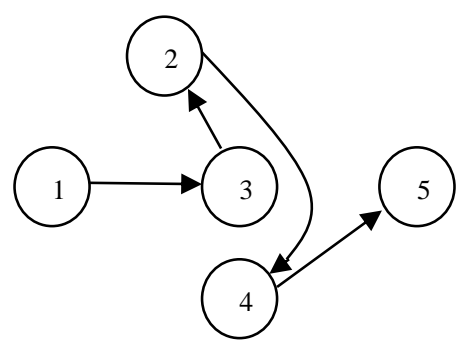

Figure 4 . The figure of the third route 


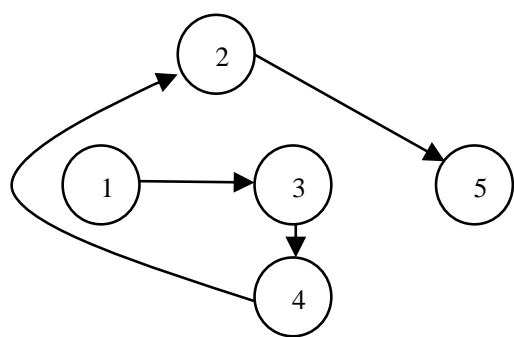

Figure 5. The figure of the fourth route

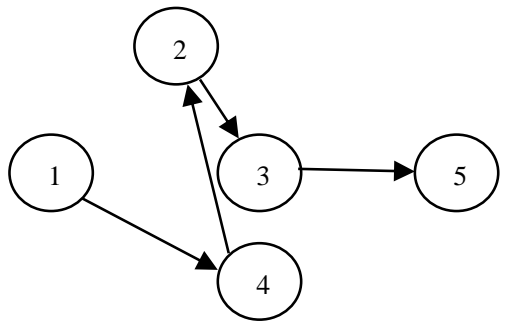

Figure 6. The figure of the fifth route

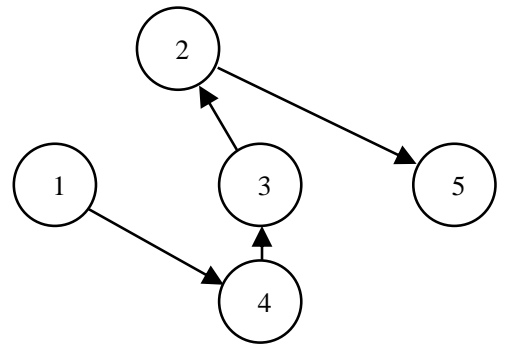

Figure 7. The figure of the sixth route

The demand parameter of each node is showed in Table 1. Service time means the time in which the vehicle must stay at customer points to provide service. Capacity demand refers to total quantity of goods demanded by the customer. Minimum arrival probability requires that the vehicle must reach the node at higher than the probability. We can conclude that the parameters of service time and capacity demand must be zero at the depot node and its mirror node, and the minimum arrival probability must be one at the depot node in any model.

Table 2 to Table 4 are arrival probability between nodes within different permit travelling time on the road. The arrival probability increases with the permit travelling time increases. The arrival probability will be zero if the road directly linked the two nodes is impassable.

Table 1. Demand parameter of each node

\begin{tabular}{|c|c|c|c|}
\hline Node & Service time (hour) & Capacity demand (ton) & Minimum arrival probability \\
\hline 1 & 0 & 0 & 1 \\
\hline 2 & 3 & 15 & 0.8 \\
\hline 3 & 2 & 20 & 1 \\
\hline 4 & 3 & 18 & 0.85 \\
\hline 5 & 0 & 0 & 0.7 \\
\hline
\end{tabular}

\begin{tabular}{|c|c|c|c|c|c|}
\multicolumn{7}{|c|}{ Table 2. Arrival probability between nodes within 2 hours } \\
\hline \multirow{2}{*}{ Node } & 1 & 2 & 3 & 4 & 5 \\
\hline 1 & & & & & \\
\hline 2 & 1 & 0.6 & 0.5 & 0.55 & 0 \\
\hline 3 & 0.6 & 1 & 0.6 & 0.3 & 0.45 \\
\hline 4 & 0.5 & 0.6 & 1 & 0.8 & 0.6 \\
\hline 5 & 0.55 & 0.3 & 0.8 & 1 & 0.5 \\
\hline
\end{tabular}


Table 3. Arrival probability between nodes within 4 hours

\begin{tabular}{|c|c|c|c|c|c|}
\hline & & & & \\
& & \\
\end{tabular}

Table 4. Arrival probability between nodes within 6 hours

\begin{tabular}{|c|c|c|c|c|c|}
\hline & \multicolumn{2}{|c|}{ Table 4. Arrival probability between nodes within 6 hours } & & \\
\\
\hline
\end{tabular}

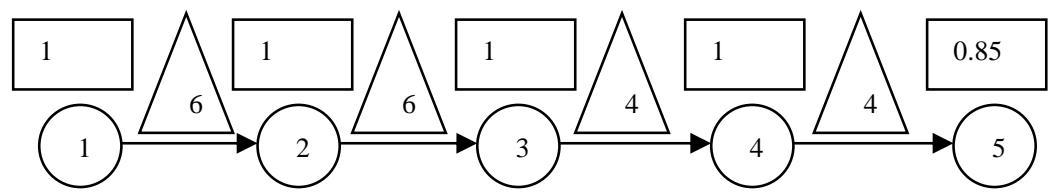

20

Figure 8 . The optimal solution of the first route

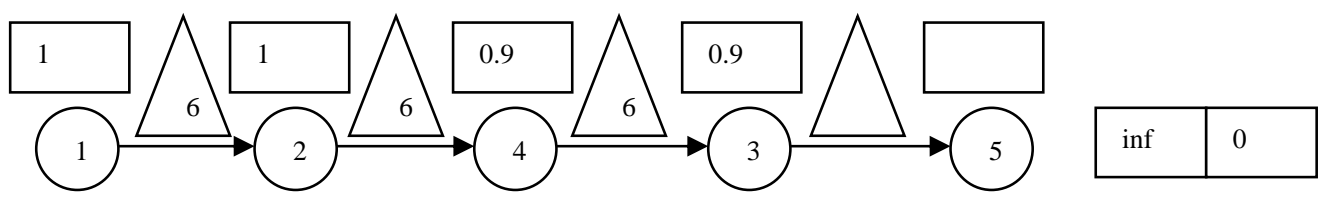

Figure 9. The optimal solution of the second route
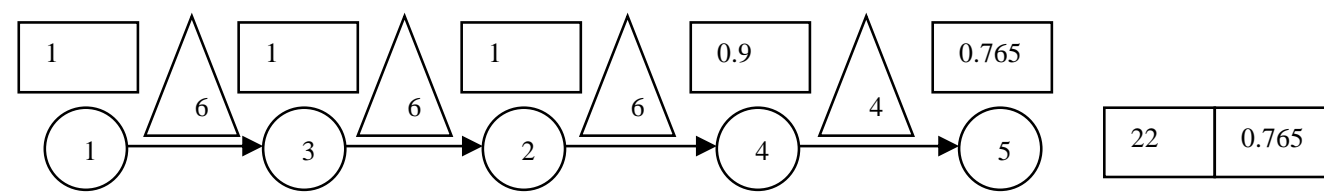

Figure 10. The optimal solution of the third route

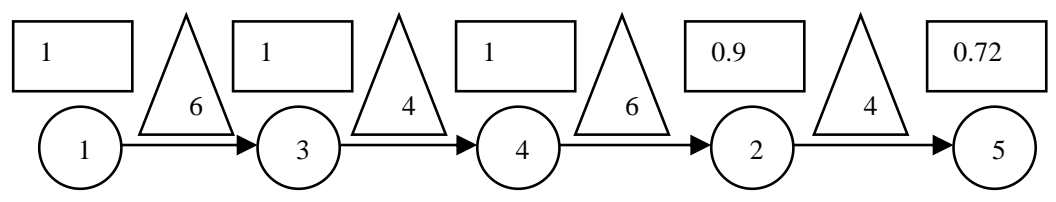

\begin{tabular}{|l|l|}
\hline 20 & 0.72 \\
\hline
\end{tabular}

Figure 11. The optimal solution of the fourth route
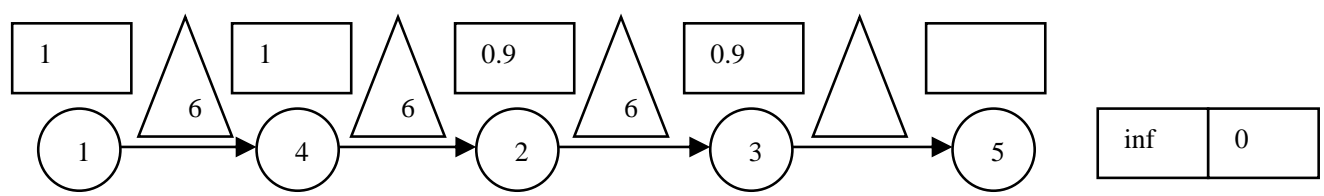

Figure 12. The optimal solution of the fifth route 


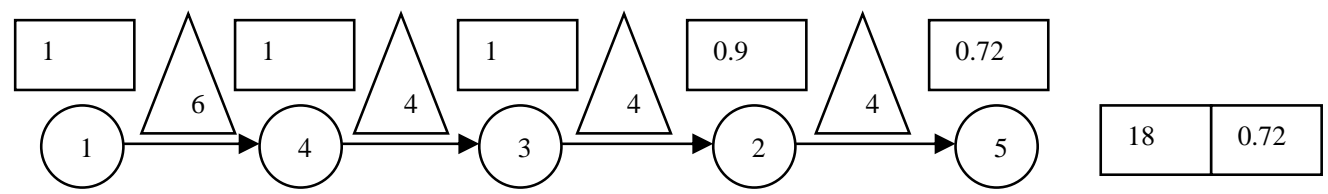

Figure 13. The optimal solution of the sixth route

There are several steps for calculating the optimal solution in small and simple models. First list all the solutions, second calculate the time spent on the road and arrival probability of each node according to the probability requirements and the tables of arrival probability between nodes within different time from front to back. In other words, obtain the time spent on the road between two nodes according to the node arrival probability requirement. Some solutions fail to meet the arrival probability requirements, which are proved to be not feasible solutions. Finally, all feasible solutions must satisfy the requirements of all nodes arrival probability. The shortest time spent on the road is the optimal solution.

Figure 8 to Figure 13 show the calculation process. We can find out the solution 1-2-4-3-5 and 1-4-2-3-5 are not feasible solutions because of the arrival probability of node 3 in both solutions can only reach 0.9 and the requirement of the arrival probability of node 3 is 1 . The global optimal solution is the sixth route 1-4-3-2-5. The total time spent on the road equals 18 , which is the least compared with other solutions, and the total service time equals 8 , according to Table 1 . Thus, the total travel time equals 26 and the total capacity of the vehicle is 53 ton according to Table 1.

Table 5. The important symbols and their brief meaning

\begin{tabular}{|c|c|}
\hline Symbols & Brief meaning \\
\hline$a_{i}$ & Requested arrival probability of node $\mathrm{i}$ \\
\hline$b_{k}$ & Capacity of the vehicle $\mathrm{k}$ \\
\hline$d_{i}$ & Capacity demand of node $i$ \\
\hline$s_{i}$ & Service time at node $\mathrm{i}$ \\
\hline$L_{i}$ & The earliest arrival time at node $\mathrm{i}$ \\
\hline$U_{i}$ & The latest arrival time at node $\mathrm{i}$ \\
\hline$y_{i}$ & Arrival probability of a vehicle at node $\mathrm{i}$ \\
\hline$t_{i}$ & Vehicle departure time from node $\mathrm{i}$ \\
\hline$w$ & Travel time of each arc linked nodes \\
\hline$M$ & Time intervals sets \\
\hline$m$ & $m \in M, m$ is one of the time intervals \\
\hline$K$ & The total number of vehicles performing transport tasks \\
\hline$P$ & Probabilities sets \\
\hline$\lambda_{1}$ & Important factors of vehicle quantity \\
\hline$\lambda_{2}$ & Important factors of total time \\
\hline$V_{d}$ & Depot node, generally $V_{d}=\{1\}$ \\
\hline$V_{c}$ & Customer nodes, generally $V_{c}=\{2, \ldots, n\}$ \\
\hline$V_{e}$ & $\begin{array}{l}\text { The mirror image of depot, the ending node of vehicles, } \\
\text { generally } V_{e}=\{n+1, \ldots n+K\}\end{array}$ \\
\hline$V$ & All nodes, generally $V=\{1,2, \ldots, n, n+1, \ldots, n+K\}$ \\
\hline$x_{i j}^{m p}$ & $\begin{array}{l}\text { Binary variable; equals } 1 \text { if a vehicle traverse directly from node } \\
\mathrm{i} \text { to node } \mathrm{j} \text {, vehicle departs from node } \mathrm{i} \text { during time interval } \mathrm{m} \\
\text { and arrives to node } \mathrm{j} \text { in time } t_{i}+w(i, j)_{p}^{m} \text { with probability } \mathrm{p} \text {, } \\
\text { otherwise it equals } 0\end{array}$ \\
\hline$\Omega_{l}^{k}$ & $\begin{array}{l}\text { Binary variable; equals } 1 \text { if the node } \mathrm{i} \text { is served by the kth } \\
\text { vehicle, else it equals } 0\end{array}$ \\
\hline
\end{tabular}

\section{The mathematical model}

The time probabilistic vehicle routing problem (TPVRP) is a problem of serving all customers by using minimal number of vehicles and total time. The total time is the sum of the total service time and total travel time [13], while the total service time is a fixed value in any feasible solution, and the total travel time includes vehicles travel time on the road and wait time on the road or customer service points. So, the total time mainly refers to the total travel time. There are two targets in the 
object function, using the minimum number of vehicles and minimum time, the degree of importance to object function between them is governed by coefficients. The input of the problem consists of probability requests of customers, position of customers and the depot, capacity demand and service time of customers. The service time here does not include the waiting time. The input of the problem also consists several tables, which show the arrival probability between two nodes within different time interval. Each vehicle has a given load capacity which means the weight of the goods on the vehicle must not exceeds the value. All vehicles leave from the depot at the beginning and return back to the same depot in the end.

The outputs of the problem are the quantity of vehicles and the routes for each vehicle. A route is a sequence of customer locations. The sum of all customers demand on the route is less than the maximum load capacity of the vehicle. We discuss the problem by using graph theory [7]. A weighted directed multigraph $\mathrm{G}(\mathrm{V}, \mathrm{E}, \mathrm{w})$ is drawn, $\mathrm{V}$ is set of nodes, $\mathrm{E}$ is set of arcs and $\mathrm{w}$ is travel time of each arc linked the two nodes[4]. More symbols and their brief meaning are listed in table 5 .

$$
Z=\min \left(\lambda_{1} K+\lambda_{2} \cdot \sum_{k=1}^{K} t_{n+k}\right)
$$

\section{Subject to}

$$
\begin{gathered}
\sum_{\substack{i=1 \\
i \neq j}}^{n} \sum_{m \in M} \sum_{p \in P} x_{i j}^{m p}=1 \quad \forall j \in V \\
\sum_{\substack{j=2 \\
j \neq i}}^{n+K} \sum_{m \in M} \sum_{p \in P} x_{i j}^{m p}=1 \quad \forall i \in V_{c} \\
\sum_{j=2}^{n+K} \sum_{m \in M} \sum_{p \in P} x_{1}^{m p}=K \\
\forall i, j \in V, \forall m \in M, \forall p \in P \\
x_{i j}^{m p} \in\{0,1\} \quad \forall i \in V \\
L_{i} \leq t_{i}-s_{i} \leq U_{i} \quad \forall i \in V_{c} \\
\sum_{i=2}^{n} d_{i} \cdot \Omega_{i}^{k} \leq b_{k} \quad \forall i \in V_{c} \\
y_{i} \geq a_{i} \quad \forall i \in V_{c} \\
t_{1}=0 \\
\end{gathered}
$$

The objective function (1) minimizes the sum of total number of used vehicles and time, $\lambda_{1}$ and $\lambda_{2}$ are influence factors. Constraints (2) and (3) ensure that each customer will be served exactly once. Constraint (4) ensures that each vehicle starts from the depot (node 1) and will return to the mirror node of depot in the end. Constraint (5) shows that there are only two results whether the vehicle has traveled from node i to j. Inequality (6) ensures that residence time of the 
vehicle is within the time window specified by the customer. Customers also cannot forward the time windows, which will be easy compared with model with customer time windows. Inequality (7) ensures that the total demand of the route is not exceed the load capacity of the vehicle. Inequality (8) means that the arrival probability of each node must greater than or equal to the requested arrival probability in every feasible route, which is the core of searching algorithm. Constraints (9)(11) give the range of several variables.

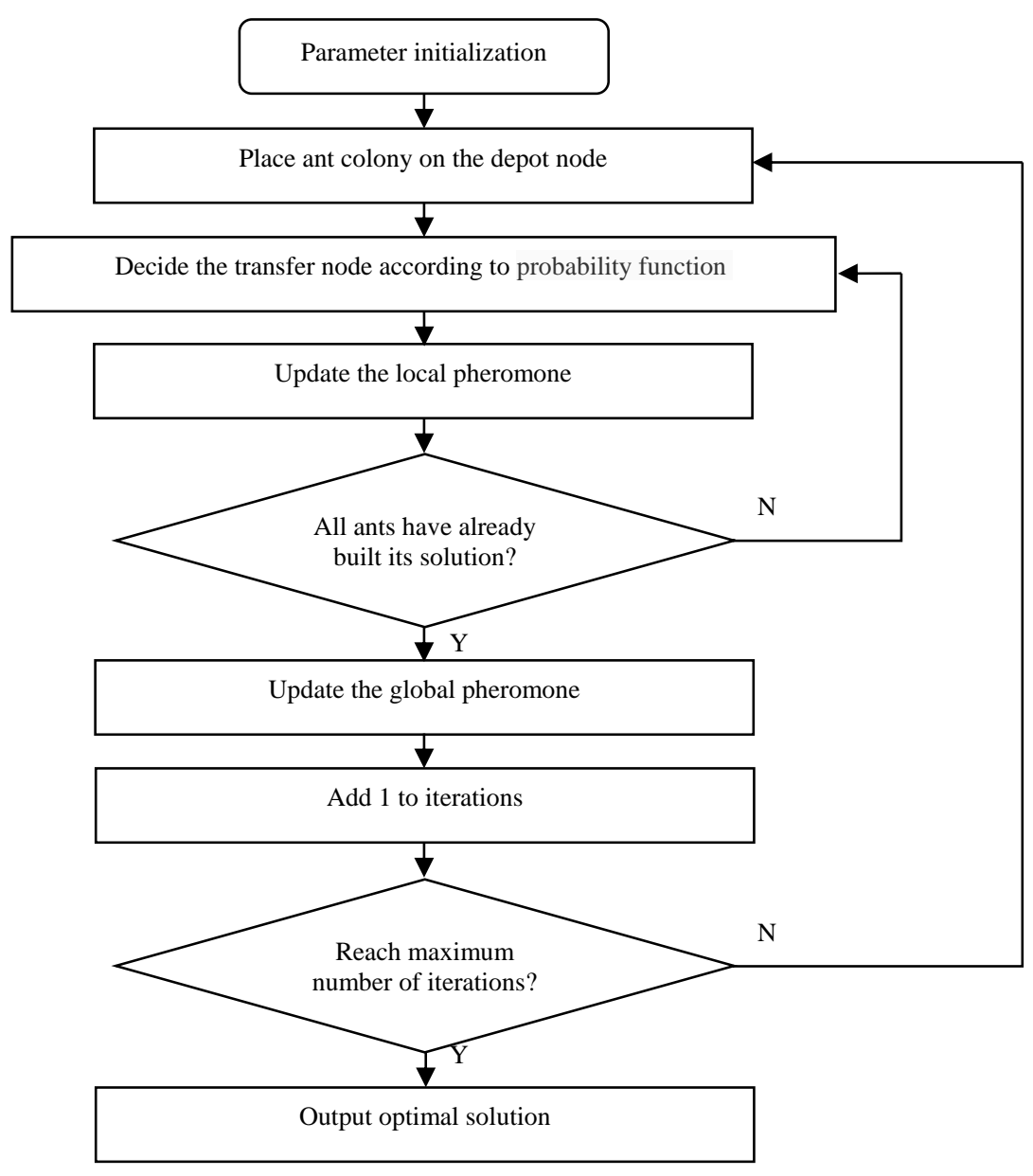

Figure 14. The flow chart of classical ant colony optimization algorithm

\section{The solution using ant colony optimization algorithm}

It is impossible to adopt artificial calculation in complex models because of the highly complexity. We recommend the ant colony optimization algorithm (ACO) for solving the problem in limited time according to the characters of the model [5].

The ant colony optimization algorithm was based on observations of the real ant colonies searching for food. Ants are capable of solving complex problems through cooperation, such as searching for food, and they can find a good path even without eyes between their nest and their destination [6]. The ants will deposit pheromone on the paths they walk, and subsequent ants can then choose the next node on the route according to the density of pheromone. Once all the ants have finished their tours, the amount of pheromone on the routes will have been modified [3].

The ant colony optimization algorithm was thus summarized and designed to solve optimization problems by simulating the ant behavior. The process of modifying the amount of pheromone on a route is called the updating rule [8], which is designed to give more pheromone to a better path and the pheromone volatilization mechanism was also designed. The algorithm has been proved that it can solve difficult NP-hard problems effectively, such as the Traveling Salesman Problem (TSP), Quadratic Assignment Problem (QAP), Vehicle Routing Problem (VRP), Job Schedule Problem (JSP) et al. 


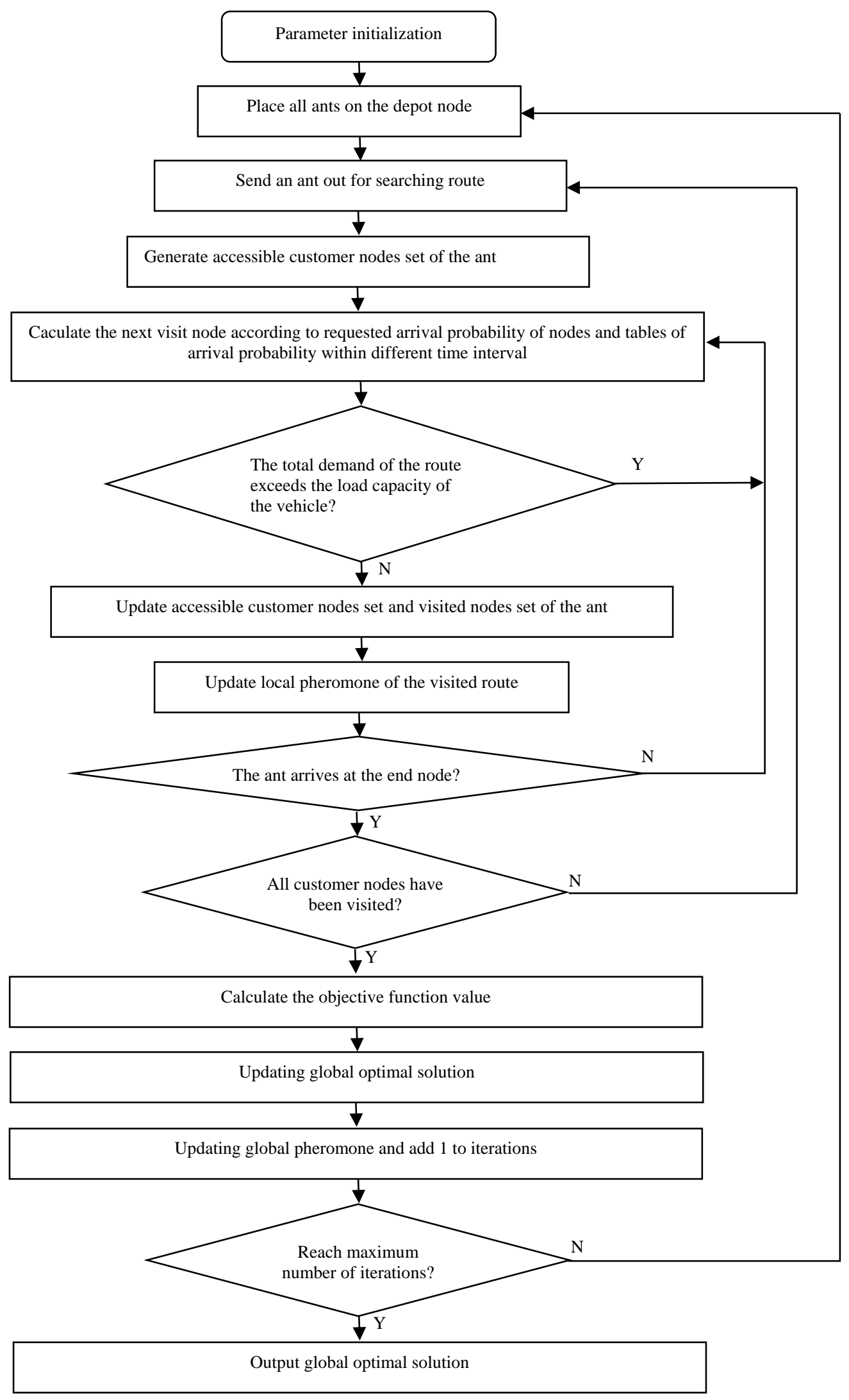

Figure 15. The solution using ant colony optimization algorithm 
The probability for the kth ant to change from its current node $\mathrm{j}$ to one of the next nodes $\mathrm{i}$ are showed in the formula (12)-(16). $R_{k}(j)$ is the set of nodes which have not been visited by the node. $\tau(j, n)$ is the pheromone on the edge from node $\mathrm{j}$ to node $\mathrm{n} . \eta(j, n)$ is heuristic information between node $\mathrm{j}$ and node $i$. $\partial$ and $\beta$ are impact factors of pheromone and heuristic information respectively. In addition, parameter $q$ is a random value uniformly distributed between 0 and $1, q_{0}$ is a control parameter, which can avoid algorithm falling into local optimum quickly. When an ant chooses an edge between two nodes $\mathrm{j}$ and $\mathrm{i}$, it will immediately update the pheromone of the edge, which is called local pheromone update rule. Formula (13) gives its specific definition. Parameter $\rho$ is called volatilization parameter, which simulates the actual situation in the real world. In the ACO process, when an edge has more pheromone, ants will select it with a higher probability. Local pheromone update rule will help reduce the pheromone of good edges currently, making ants do not always choose the same edges, and avoiding the risk of choosing a local optimum solution in the search process. After all the ants have completed their tours, the pheromone on the best route will be updated according to formula (15)-(16), which are called global pheromone update rule.

The ant colony optimization algorithm chooses nodes according to probability which is suitable to solve the problem. Figure 14 is the flow chart of classical ant colony optimization algorithm, and Figure 15 is the solution plan using ant colony optimization algorithm. Ants will search all the nodes step by step to form solutions, and update the optimal solutions continuous, finally obtain the optimal solution in limited time.

$$
\begin{gathered}
P= \begin{cases}\arg _{n \in R_{k}(j)}^{\max }\left[\tau(j, n)^{\alpha} \times \eta(j, n)^{\beta}\right] & q \leq q_{0} \\
P_{k}(j, i) & q>q_{0}\end{cases} \\
P_{k}(j, i)= \begin{cases}\frac{\tau(j, i)^{\alpha} \times \eta(j, i)^{\beta}}{\sum_{n \in R_{k}(j)}\left[\tau(j, n)^{\alpha} \times \eta(j, n)^{\beta}\right]} & i \in R_{k}(j) \\
0 & \text { otherwise }\end{cases} \\
\tau_{(t+1)}(j, i)=(1-\rho) \times \tau_{t}(j, i)+\rho \times \tau_{0}
\end{gathered}
$$

\section{Conclusions}

The paper proposes a new variant of vehicle routing problem and analyzes the characteristics of the problem. The Ant colony algorithm is suggested to solve the problem, and the concrete solving steps are given. The ant colony algorithm can solve the complex problem efficiently.

\section{Acknowledgements}

This work was supported by 2017 natural science research project of Anhui University (KJ2017A429), and supported by 2017 Anhui College of Humanities and social science research project(SK2017A0437), also supported by robot virtual simulation experimental teaching center project of Anhui university of finance \& economics (acxnfz201701)

\section{References}

1. B. Bedregal, R. Reiser, H. Bustince, C. L. Molina, V. Torra, "Aggregation functions for typical hesitant fuzzy elements and the action of automorphisms", Information Sciences, vol. 255, no. 1, pp. 82-99, 2014.

2. N. Chen, Z. S. Xu, M. M. Xia, "Interval-valued hesitant preference relations and their applications to group decision making", Knowledge-Based Systems, vol. 37, no. 2, pp. 528-540, 2013.

3. B. Farhadinia, "A theoretical development on the entropy of interval-valued fuzzy sets based on the intuitionistic distance and its relationship with similarity measure", Knowledge-Based Systems, vol. 39, no. 2, pp. 79-84, 2013. 
4. Z. J. Fu, F. G. Huang, K. Ren, J. Weng, C. Wang, "Privacy-preserving smart semantic search based on conceptual graphs over encrypted outsourced data", IEEE Transactions on Information Forensics and Security, vol. 12, no.8, pp. 1874-1884, 2017.

5. Z. J. Fu, K. Ren, J. G. Shu, X. M. Sun, F. X. Huang, "Enabling personalized search over encrypted outsourced data with efficiency improvement", IEEE Transactions on Parallel and Distributed Systems, vol. 27, no. 9, pp. 2546-2559, 2016.

6. Z. J. Fu, X. L. Wu, C. W. Guan, X. M. Sun, K. Ren, "Toward efficient multi-keyword fuzzy search over encrypted outsourced data with accuracy improvement", IEEE Transactions on Information Forensics and Security, vol. 11, no. 12, pp. 2706-2716, 2016.

7. H. C. Liao, Z. S. Xu, "A VIKOR-based method for hesitant fuzzy multi-criteria decision making", Fuzzy Optimization \& Decision Making, vol. 12, no. 4, pp. 373-392, 2013.

8. H. C. Liao, Z. S. Xu, X. J. Zeng, "Distance and similarity measures for hesitant fuzzy linguistic term sets and their application in multi-criteria decision making", Information Sciences, vol. 271, no. 3, pp. 125-142, 2014.

9. H. C. Liao, Z. S. Xu, X. J. Zeng, J. M. Merigó, "Qualitative decision making with correlation coefficients of hesitant fuzzy linguistic term sets", Knowledge-Based Systems, vol. 76, no. 1, pp. 127-138, 2015.

10. J. J. Peng, J. Q. Wang, X. H. Wu, H. Y. Zhang, X. H. Chen, "The fuzzy cross-entropy for intuitionistic hesitant fuzzy sets and their application in multi-criteria decision-making”, International Journal of Systems Science, vol. 46, no. 13, pp. 1-16, 2014.

11. H. Wang, "Extended hesitant fuzzy linguistic term sets and their aggregation in group decision making", International Journal of Computational Intelligence Systems, vol. 8, no. 1, pp. 14-33, 2015.

12. J. Q. Wang, Z. Q. Han, H. Y. Zhang, "Multi-criteria group decision-making method based on intuitionistic interval fuzzy information", Group Decision and Negotiation, vol. 23, no. 4, pp. 715-733, 2014.

13. J. Q. Wang, D. D. Wang, H. Y. Zhang, X. H. Chen, "Multi-criteria outranking approach with hesitant fuzzy sets", OR Spectrum, vol. 36, no. 4, pp. 1001-1019, 2014.

Ming Fu graduated from the School of Information Technology, Wuhan University of Technology, for the degree Master. $\mathrm{He}$ is a teacher of Anhui University of Finance \& Economics. His current research interests include artificial intelligence and machine learning, and intelligent information processing.

Jian Zhou is a teacher of Anhui University of Finance \& Economics. His research interests include complex system modeling and big data analysis.

Lifang Wang is a teacher of Anhui University of Finance \& Economics. Her research interests include intelligent logistics and network congestion control. 